\title{
Algebras of approximation sequences: Fredholm theory in fractal algebras
}

\author{
by \\ StefFen Roch (Darmstadt)
}

\begin{abstract}
The present paper is a continuation of [5, 7] where a Fredholm theory for approximation sequences is proposed and some of its properties and consequences are studied. Here this theory is specified to the class of fractal approximation methods. The main result is a formula for the so-called $\alpha$-number of an approximation sequence $\left(A_{n}\right)$ which is the analogue of the kernel dimension of a Fredholm operator.
\end{abstract}

1. Introduction. Let $H$ be a complex Hilbert space, $L(H)$ the $C^{*}$ algebra of bounded linear operators on $H$, and $K(H)$ the ideal of compact linear operators on $H$. Further let $\left(P_{n}\right)$ be a sequence of orthogonal projections on $H$ which converges strongly to the identity operator $I$ on $H$ :

$$
\text { s- } \lim P_{n}=I \Leftrightarrow P_{n} x \rightarrow x \text { for all } x \in H,
$$

and assume that $\operatorname{dim} \operatorname{Im} P_{n}=n$. Thus, $\operatorname{Im} P_{n}$ and $L\left(\operatorname{Im} P_{n}\right)$ can be identified with the linear space $\mathbb{C}^{n}$ and with the algebra $\mathbb{C}^{n \times n}=L\left(\mathbb{C}^{n}\right)$, respectively. The $n \times n$ identity matrix will be denoted by $I_{n}$.

Let $A \in L(H)$. An approximation method for $A$ is a sequence $\left(A_{n}\right)$ of matrices $A_{n} \in \mathbb{C}^{n \times n}$ such that $A_{n} P_{n} \rightarrow A$ and $A_{n}^{*} P_{n} \rightarrow A^{*}$ strongly as $n \rightarrow \infty$. This method converges if the equations

$$
A_{n} x^{(n)}=P_{n} y
$$

have unique solutions $x^{(n)} \in \operatorname{Im} P_{n}$ for all sufficiently large $n$ and all right hand sides $y \in H$, and if these solutions converge in the norm of $H$ to a solution of the equation

$$
A x=y .
$$

By the Banach-Steinhaus theorem, the method $\left(A_{n}\right)$ for $A$ is applicable if and only if the sequence $\left(A_{n}\right)$ is stable in the sense that the matrices $A_{n}$ are invertible for all sufficiently large $n$ and

$$
\sup \left\|A_{n}^{-1}\right\|<\infty \text {. }
$$

2000 Mathematics Subject Classification: Primary 46N40; Secondary 65R20. 
For another characterization of stability, introduce the set $\mathcal{F}$ of all bounded sequences $\left(A_{n}\right)$ of matrices $A_{n} \in \mathbb{C}^{n \times n}$. Provided with elementwise operations and the supremum norm, this set becomes a $C^{*}$-algebra with identity element $\left(I_{n}\right)$, and the set $\mathcal{G}$ of all sequences $\left(A_{n}\right) \in \mathcal{F}$ with $\lim \left\|A_{n}\right\|=0$ forms a closed two-sided ideal in $\mathcal{F}$. A Neumann series argument shows that the sequence $\left(A_{n}\right) \in \mathcal{F}$ is stable if and only if its coset $\left(A_{n}\right)+\mathcal{G}$ is invertible in the quotient algebra $\mathcal{F} / \mathcal{G}$.

The role of the algebra $\mathcal{F} / \mathcal{G}$ in numerical analysis can be compared with the role of the algebra $L(H)$ in operator theory. In this sense, the stable sequences in $\mathcal{F}$ correspond to the invertible operators on $H$. For operators, there is a useful generalization of the notion of invertibility: one considers operators $A \in L(H)$ which are almost invertible in the sense that both their kernel $\operatorname{Ker} A$ and their cokernel Coker $A:=H / \operatorname{Im} A$ have finite dimension. Operators with this property are called Fredholm operators, and the quantity ind $A:=\operatorname{dim} \operatorname{Ker} A-\operatorname{dim}$ Coker $A$ is referred to as the index of $A$.

In $[7,5]$ an analogous notion of a Fredholm approximation sequence is introduced and studied. The kernel dimension condition for a Fredholm operator is replaced by the following condition for a Fredholm sequence $\left(A_{n}\right) \in \mathcal{F}$ : There is an $\varepsilon>0$ and a fixed number $k$ such that at most $k$ singular values of $A_{n}$ are less than $\varepsilon$ for all sufficiently large $n$. The smallest possible number $k$ with this property is the analogue of the kernel dimension for the Fredholm sequence $\left(A_{n}\right)$, and we call it the $\alpha$-number of that sequence. One can also define the index of a Fredholm sequence in a natural way, but it turns out that this index is always zero. This is a consequence of the fact that the approximation operators $A_{n}$ which we consider act on finite-dimensional spaces. Exact definitions will be given in Section 2.

So the most important quantity related to a Fredholm sequence seems to be its $\alpha$-number. In [7], we derived a simple and practicable formula to compute this number for large classes of concrete approximation sequences. (In fact, for all sequences which lie in a standard algebra in the terminology of [7]. This class includes, e.g., projection methods with spline ansatz functions for singular integral operators or quadrature formula methods for Mellin operators, to mention only a few.) It has been one goal in [5] to generalize this formula to the class of all fractal approximation methods. This class has been introduced in $[6,4]$. Roughly speaking, a sequence $\left(A_{n}\right)$ is fractal (or self-similar) if $\left(A_{n}\right)$ can be completely reconstructed from each of its infinite subsequences up to a sequence tending to zero in norm. The precise definition is in Section 3. The class of fractal sequences includes all sequences in standard algebras.

For the formula for the $\alpha$-numbers derived in [7] one needs an additional hypothesis which is satisfied for all sequences in standard algebras but not for all fractal sequences. In the present paper, we will set forth a specification 
of the general Fredholm theory (including a formula for the $\alpha$-numbers) to fractal approximation methods without any additional (and hard to check) hypotheses (Section 4).

For applications of this Fredholm theory to the analysis of concrete approximation methods we refer to the already mentioned papers $[7,8,5]$ and to the textbook [2]. Nice applications to a special class of approximation sequences (the finite section method for Toeplitz operators) are in [1].

2. Compact and Fredholm sequences. In this section we recall the definition of a compact and a Fredholm approximation sequence from [5], and we derive some characterizations of compact sequences which are basic in what follows.

\subsection{The ideal of compact sequences}

Sequences of rank one matrices. Let $\mathcal{K}$ be the smallest closed ideal of $\mathcal{F}$ which contains all sequences of matrices of rank $\leq 1$. The product of such a sequence with another sequence in $\mathcal{F}$ is again a sequence of matrices of rank $\leq 1$. Hence, the set $\mathcal{K}_{0}$ of all finite sums of sequences matrices of rank $\leq 1$ forms an ideal (in general, non-closed) of $\mathcal{F}$ whose closure is just the ideal $\mathcal{K}$. Consequently, a sequence $\left(A_{n}\right) \in \mathcal{F}$ belongs to $\mathcal{K}$ if and only if, for every $\varepsilon>0$, there is a sequence $\left(K_{n}\right) \in \mathcal{F}$ such that

$$
\sup _{n}\left\|A_{n}-K_{n}\right\|<\varepsilon \text { and } \sup _{n} \operatorname{dim} \operatorname{Im} K_{n}<\infty .
$$

We refer to the elements of $\mathcal{K}$ as compact sequences. The role of the ideal $\mathcal{K}$ of compact sequences in numerical analysis can be compared with the role of the ideal of compact operators in operator theory.

Characterization of compact sequences via singular values. Given a sequence $\left(K_{n}\right) \in \mathcal{F}$, denote by

$$
0 \leq \sigma_{1}^{(n)} \leq \sigma_{2}^{(n)} \leq \ldots \leq \sigma_{n}^{(n)}
$$

the singular values of $K_{n}$, i.e. the non-negative square roots of the eigenvalues of $K_{n}^{*} K_{n}$. Further set $\Sigma_{k}^{(n)}:=\sigma_{n-k+1}^{(n)}$ for $k=1, \ldots, n$. Clearly, $\left\|K_{n}\right\|=\sigma_{n}^{(n)}=\Sigma_{1}^{(n)}$.

Theorem 2.1. A sequence $\left(K_{n}\right) \in \mathcal{F}$ is compact if and only if

$$
\lim _{k \rightarrow \infty} \sup _{n \geq k} \Sigma_{k}^{(n)}=0 .
$$

Proof. Let $\left(K_{n}\right) \in \mathcal{F}$ satisfy (3), and let (with unitary matrices $U_{n}, V_{n}$ )

$$
K_{n}=U_{n} \operatorname{diag}\left(\Sigma_{1}^{(n)}, \ldots, \Sigma_{n}^{(n)}\right) V_{n}^{*}
$$


be the singular value decomposition of $K_{n}$. For every $k, n \geq 1$, set

$$
K_{n}^{(k)}:= \begin{cases}U_{n} \operatorname{diag}\left(\Sigma_{1}^{(n)}, \ldots, \Sigma_{k-1}^{(n)}, 0, \ldots, 0\right) V_{n}^{*} & \text { if } 1<k \leq n \\ 0 & \text { if } 1=k \leq n \\ K_{n} & \text { if } n<k .\end{cases}
$$

Then, for all $k, n \geq 1$,

$$
\begin{aligned}
\left\|K_{n}-K_{n}^{(k)}\right\| \\
= \begin{cases}\left\|U_{n} \operatorname{diag}\left(0, \ldots, 0, \Sigma_{k}^{(n)}, \ldots, \Sigma_{n}^{(n)}\right) V_{n}^{*}\right\|=\Sigma_{k}^{(n)} & \text { if } 1<k \leq n \\
\left\|K_{n}\right\|=\Sigma_{1}^{(n)} & \text { if } 1=k \leq n \\
0 & \text { if } n<k .\end{cases}
\end{aligned}
$$

Hence,

$$
\left\|\left(K_{n}\right)_{n \geq 1}-\left(K_{n}^{(k)}\right)_{n \geq 1}\right\|_{\mathcal{F}}=\sup _{n \geq k} \Sigma_{k}^{(n)}
$$

which together with hypothesis (3) implies

$$
\lim _{k \rightarrow \infty}\left\|\left(K_{n}\right)_{n \geq 1}-\left(K_{n}^{(k)}\right)_{n \geq 1}\right\|_{\mathcal{F}}=\lim _{k \rightarrow \infty} \sup _{n \geq k} \Sigma_{k}^{(n)}=0 .
$$

Thus, the sequence $\left(K_{n}\right)$ is the limit as $k \rightarrow \infty$ of the sequences $\left(K_{n}^{(k)}\right)_{n \geq 1}$. Since $\operatorname{dim} \operatorname{Im} K_{n}^{(k)} \leq k-1$, each $\left(K_{n}^{(k)}\right)_{n \geq 1}$ lies in $\mathcal{K}_{0}$, and so $\left(K_{n}\right)$ is compact.

Let now $\left(K_{n}\right)$ be a compact sequence. Then $\left(\sup _{n \geq k} \Sigma_{k}^{(n)}\right)_{k \geq 1}$ is decreasing and bounded below (by zero) and, hence, convergent. Assume that its limit is positive. Then there is a $C>0$ such that

$$
\sup _{n \geq k} \Sigma_{k}^{(n)} \geq C \quad \text { for all } k \geq 1 \text {. }
$$

Hence, for each $k \geq 1$, there is an $n_{k} \geq k$ such that

$$
\Sigma_{k}^{\left(n_{k}\right)} \geq C \quad \text { for all } k \geq 1 \text {. }
$$

Further, since $\left(K_{n}\right)$ is compact, there is a sequence $\left(R_{n}\right) \in \mathcal{F}$ with

$$
\sup _{n} \operatorname{dim} \operatorname{Im} R_{n}<\infty \quad \text { and } \sup _{n}\left\|K_{n}^{*} K_{n}-R_{n}^{*} R_{n}\right\|<C
$$

(cf. (1)). Set $r:=\sup _{n} \operatorname{dim} \operatorname{Im} R_{n}$ and choose $k>r$. Thus, in particular,

$$
\left\|K_{n_{k}}^{*} K_{n_{k}}-R_{n_{k}}^{*} R_{n_{k}}\right\|<C \text {. }
$$

Let now

$$
K_{n_{k}}^{*} K_{n_{k}}=U_{n_{k}} \operatorname{diag}\left(\Sigma_{1}^{\left(n_{k}\right)}, \ldots, \Sigma_{n_{k}}^{\left(n_{k}\right)}\right) U_{n_{k}}^{*}
$$

with a unitary matrix $U_{n_{k}}$, and set

$$
P_{k}:=\operatorname{diag}(1, \ldots, 1,0, \ldots, 0)
$$

with $k$ ones and $n_{k}-k$ zeros. Then, obviously, the matrix

$$
P_{k} U_{n_{k}}^{*} K_{n_{k}}^{*} K_{n_{k}} U_{n_{k}} P_{k}=\operatorname{diag}\left(\Sigma_{1}^{\left(n_{k}\right)}, \ldots, \Sigma_{k}^{\left(n_{k}\right)}, 0, \ldots, 0\right)
$$


is invertible on $\operatorname{Im} P_{k}$, and

$$
\left\|\left(\left.P_{k} U_{n_{k}}^{*} K_{n_{k}}^{*} K_{n_{k}} U_{n_{k}} P_{k}\right|_{\operatorname{Im} P_{k}}\right)^{-1}\right\|=\max \left\{\frac{1}{\Sigma_{1}^{\left(n_{k}\right)}}, \ldots, \frac{1}{\Sigma_{k}^{\left(n_{k}\right)}}\right\}=\frac{1}{\Sigma_{k}^{\left(n_{k}\right)}},
$$

whence

$$
\left\|\left(\left.P_{k} U_{n_{k}}^{*} K_{n_{k}}^{*} K_{n_{k}} U_{n_{k}} P_{k}\right|_{\operatorname{Im} P_{k}}\right)^{-1}\right\|^{-1}=\Sigma_{k}^{\left(n_{k}\right)} \geq C
$$

due to (4). Since, by (5),

$$
\left\|P_{k} U_{n_{k}}^{*} K_{n_{k}}^{*} K_{n_{k}} U_{n_{k}} P_{k}-P_{k} U_{n_{k}}^{*} R_{n_{k}}^{*} R_{n_{k}} U_{n_{k}} P_{k}\right\|<C,
$$

a Neumann series argument yields the invertibility of $P_{k} U_{n_{k}}^{*} R_{n_{k}}^{*} R_{n_{k}} U_{n_{k}} P_{k}$, considered as an operator on $\operatorname{Im} P_{k}$. This implies that

$$
\operatorname{dim} \operatorname{Im} R_{n_{k}}^{*} R_{n_{k}} \geq \operatorname{dim} \operatorname{Im} P_{k}=k,
$$

which contradicts our assumption. Hence, the sequence $\left(\sup _{n \geq k} \Sigma_{k}^{(n)}\right)_{k \geq 1}$ cannot have a positive limit, i.e. $\left(K_{n}\right)$ satisfies $(3)$.

Corollary 2.2. Let $\left(K_{n}\right) \in \mathcal{K}$. Then $\lim _{n \rightarrow \infty} \sigma_{k}^{(n)}$ exists and is 0 for every $k$.

Proof. Let $\varepsilon>0$. By Theorem 2.1, there is a $k_{0}$ such that $\sup _{n \geq k_{0}} \Sigma_{k_{0}}^{(n)}$ $<\varepsilon$. Then, for all $n \geq n_{0}:=k_{0}+k-1$,

$$
\sigma_{k}^{(n)}=\Sigma_{n-k+1}^{(n)} \leq \Sigma_{k_{0}}^{(n)} \leq \sup _{n \geq k_{0}} \Sigma_{k_{0}}^{(n)}<\varepsilon
$$

Further characterizations of the ideal $\mathcal{K}$. We proceed with two further characterizations of the ideal $\mathcal{K}$ which are partially based on the results of the preceding subsection. One aim of these characterizations is to show that the above definition of compact sequences is in some sense the only possible one. Indeed, suppose for a moment that we have no idea of what a compact sequence might be and that we would like to introduce an ideal of $\mathcal{F}$ which corresponds to the ideal of compact operators on a Hilbert space. The concrete approximation methods considered and mentioned in [6] suggest that the constant sequence $\left(P_{1}\right)$ where $P_{1}$ is the diagonal matrix $\operatorname{diag}(1,0, \ldots, 0)$ should be considered as compact in any case. Thus, a minimal candidate for the desired ideal of compact sequences is the smallest closed ideal of $\mathcal{F}$ which contains the sequence $\left(P_{1}\right)$. There is also a natural largest candidate: the ideal of all sequences $\left(K_{n}\right)$ such that $W\left(K_{n}\right)$ is a compact operator for every irreducible representation $W$ of $\mathcal{F}$. The characterizations below show that actually both candidates coincide with the ideal $\mathcal{K}$ defined before.

Theorem 2.3. $\mathcal{K}$ is the smallest closed ideal of $\mathcal{F}$ which contains the constant sequence $\left(P_{1}\right)$.

Proof. Let, for a moment, $\mathcal{K}^{\prime}$ stand for the smallest closed ideal of $\mathcal{F}$ which contains $\left(P_{1}\right)$. It is evident that $\mathcal{K}^{\prime} \subseteq \mathcal{K}$. For the reverse inclusion, we 
have to verify that every sequence $\left(K_{n}\right) \in \mathcal{F}$ of rank $\leq 1$ matrices belongs to $\mathcal{K}^{\prime}$.

Indeed, for such a sequence define

$$
K_{n}^{\prime}:= \begin{cases}\left\|K_{n}^{*} K_{n}\right\|^{-1} K_{n}^{*} K_{n} & \text { if } K_{n} \neq 0 \\ P_{1} & \text { if } K_{n}=0 .\end{cases}
$$

Every matrix $K_{n}^{\prime}$ is an orthogonal projection of rank one. Hence, there are unitary matrices $U_{n} \in \mathbb{C}^{n \times n}$ such that

$$
K_{n}^{\prime}=U_{n}^{*} \operatorname{diag}(1,0, \ldots, 0) U_{n}=U_{n}^{*} P_{1} U_{n} .
$$

The sequence $\left(U_{n}\right)$ is bounded and belongs therefore to $\mathcal{F}$. Hence, $\left(K_{n}^{\prime}\right)=$ $\left(U_{n}^{*}\right)\left(P_{1}\right)\left(U_{n}\right)$ belongs to $\mathcal{K}^{\prime}$, and so does $\left(K_{n}\right)$ because of

$$
\left(K_{n}\right)=\left(\lambda_{n}\right)\left(K_{n}\right)\left(K_{n}^{\prime}\right) \quad \text { with } \quad \lambda_{n}= \begin{cases}1 & \text { if } K_{n} \neq 0 \\ 0 & \text { if } K_{n}=0\end{cases}
$$

THEOREM 2.4. (a) If $\left(K_{n}\right) \in \mathcal{F}$ is a sequence of rank $\leq 1$ matrices and $(H, \pi)$ is an irreducible representation of $\mathcal{F}$, then $\pi\left(K_{n}\right)$ is an operator with rank $\leq 1$.

(b) A sequence $\left(K_{n}\right) \in \mathcal{F}$ belongs to the ideal $\mathcal{K}$ if and only if $\pi\left(K_{n}\right)$ is compact for every irreducible representation $(H, \pi)$ of $\mathcal{F}$.

Proof. Assertion (a) is proved in [5] (Proposition 2 and Theorem 3). For a proof of (b), let $\mathcal{K}^{\prime}$ stand for the set of all sequences $\left(K_{n}\right)$ in $\mathcal{F}$ such that $\pi\left(K_{n}\right)$ is compact for every irreducible representation $\pi$ of $\mathcal{F}$. The inclusion $\mathcal{K} \subseteq \mathcal{K}^{\prime}$ is an immediate consequence of (a). For the proof of the reverse inclusion, we will show that, for every sequence $\left(K_{n}\right) \in \mathcal{F} \backslash \mathcal{K}$, there is an irreducible representation $\pi$ of $\mathcal{F}$ such that $\pi\left(K_{n}\right)$ is not compact.

Let $\left(K_{n}\right) \in \mathcal{F}$ be a sequence which is not in $\mathcal{K}$, and let $\operatorname{Id}\left(K_{n}\right)$ be the smallest closed ideal of $\mathcal{F}$ which contains the sequence $\left(K_{n}\right)$. Further, let $\Lambda_{1}^{(n)} \geq \ldots \geq \Lambda_{n}^{(n)} \geq 0$ denote the eigenvalues of $K_{n}^{*} K_{n}$, and let $U_{n}$ be a unitary matrix such that

$$
U_{n}^{*} K_{n}^{*} K_{n} U_{n}=\operatorname{diag}\left(\Lambda_{1}^{(n)}, \ldots, \Lambda_{n}^{(n)}\right) .
$$

Since $\left(K_{n}\right) \notin \mathcal{K}$, Theorem 2.1 implies that $\lim _{k \rightarrow \infty} \sup _{n \geq k} \Lambda_{k}^{(n)} \neq 0$. Thus, as we checked in the proof of Theorem 2.1, there is a $C>0$ as well as a sequence $\left(n_{k}\right)_{k \geq 1}$ with $n_{k} \geq k$ such that

$$
\Lambda_{k}^{\left(n_{k}\right)} \geq C \text { for all } k \text {. }
$$

The sequence $\left(n_{k}\right)$ can be chosen strictly increasing. Indeed, suppose that we have already determined $n_{1}<\ldots<n_{k}$. Choose $k^{\prime}>n_{k}$ and a corresponding $n^{\prime} \geq k^{\prime}$ such that $\Lambda_{k^{\prime}}^{\left(n^{\prime}\right)} \geq C$, which is possible due to (6). Then $n_{k+1}:=n^{\prime}$ satisfies $n_{k+1}=n^{\prime} \geq n_{k}+1 \geq k+1$, yielding the desired strict monotonicity. 
So let $\left(n_{k}\right)$ be a strictly increasing sequence satisfying (6). Set

$$
L_{n}:=\operatorname{diag}\left(1 / \Lambda_{1}^{\left(n_{k}\right)}, \ldots, 1 / \Lambda_{k}^{\left(n_{k}\right)}, 0, \ldots, 0\right)
$$

if $n=n_{k}$ for some $k$, and $L_{n}:=0$ if $n \neq n_{k}$. The sequence $\left(L_{n}\right)$ is bounded because $\left(\Lambda_{j}^{\left(n_{k}\right)}\right)^{-1} \leq 1 / C$ for $j=1, \ldots, k$. Hence, the sequence $\left(D_{n}\right)$ with

$$
D_{n}:=L_{n} U_{n}^{*} K_{n}^{*} K_{n} U_{n}= \begin{cases}0 & \text { if } n \neq n_{k}, \\ \operatorname{diag}(1, \ldots, 1,0, \ldots, 0) & \text { if } n=n_{k},\end{cases}
$$

with $k$ ones and $n_{k}-k$ zeros belongs to the ideal $\operatorname{Id}\left(K_{n}\right)$. Then all sequences

$$
\left(D_{n} P_{n} A P_{n} D_{n}\right) \quad \text { with } A \in L\left(l^{2}\right)
$$

lie in $\operatorname{Id}\left(K_{n}\right)$, and we consider the smallest closed $C^{*}$-subalgebra $\mathcal{B}$ of $\operatorname{Id}\left(K_{n}\right)$ which contains all sequences (7). Clearly, the strong $\operatorname{limit}_{\mathrm{s}} \lim _{k \rightarrow \infty} B_{n_{k}}$ exists for every sequence $\left(B_{n}\right) \in \mathcal{B}$ and, in particular,

$$
\operatorname{sim}_{k \rightarrow \infty} D_{n_{k}} P_{n_{k}} A P_{n_{k}} D_{n_{k}}=A
$$

for all sequences of the form (7). The mapping

$$
W: \mathcal{B} \rightarrow L\left(l^{2}\right), \quad\left(B_{n}\right) \mapsto \operatorname{s}_{k \rightarrow \infty} \lim _{n_{k}},
$$

is an irreducible representation of $\mathcal{B}$ (all compact operators lie in the range of $W)$ which maps $\left(D_{n}\right)$ to the identity operator on $l^{2}$. From Proposition 4.1.8 of [3] we know that there exists an irreducible representation $\pi: \mathcal{F} \rightarrow L(H)$, a closed subspace $H_{1}$ of $H$, and a bijective isometry $U: H_{1} \rightarrow l^{2}$ such that

$$
W\left(B_{n}\right)=\left.U \pi\left(B_{n}\right)\right|_{H_{1}} U^{*} \quad \text { for all }\left(B_{n}\right) \in \mathcal{B} .
$$

The operator $\pi\left(K_{n}\right)$ cannot be compact. Indeed, suppose that it is. Then the operators $\pi\left(R_{n}\right)$ are compact for all sequences $\left(R_{n}\right) \in \operatorname{Id}\left(K_{n}\right)$, hence so are the operators $\pi\left(D_{n}\right)$. Then, by (8), $W\left(D_{n}\right)$ must be compact. This is impossible since $W\left(D_{n}\right)$ is the identity operator on $l^{2}$ as we already observed. Thus, $\left(K_{n}\right) \notin \mathcal{K}^{\prime}$, yielding the inclusion $\mathcal{K}^{\prime} \subseteq \mathcal{K}$.

2.2. Fredholm sequences. Corresponding to the ideal $\mathcal{K}$ we introduce an appropriate class of Fredholm sequences by calling a sequence $\left(A_{n}\right) \in \mathcal{F}$ Fredholm if it is invertible modulo $\mathcal{K}$ (see [5]). The following properties of Fredholm sequences are obvious.

- Every stable sequence is Fredholm.

- The adjoint of a Fredholm sequence is Fredholm.

- The product of Fredholm sequences is Fredholm.

- If $\left(A_{n}\right)$ is Fredholm and $\left(K_{n}\right) \in \mathcal{K}$, then $\left(A_{n}+K_{n}\right)$ is Fredholm.

- The set of Fredholm sequences is open in $\mathcal{F}$.

For another characterization of Fredholm sequences, let $0 \leq \sigma_{1}^{(n)} \leq \ldots \leq$ $\sigma_{n}^{(n)}$ denote the singular values of a sequence $\left(A_{n}\right) \in \mathcal{F}$. 
THEOREM 2.5. Each of the following conditions is equivalent to the Fredholmness of a sequence $\left(A_{n}\right) \in \mathcal{F}$ :

(a) There are sequences $\left(B_{n}\right) \in \mathcal{F}$ and $\left(J_{n}\right) \in \mathcal{K}$ with $\sup _{n} \operatorname{dim} \operatorname{Im} J_{n}$ $<\infty$ such that

$$
B_{n} A_{n}^{*} A_{n}=I_{n}+J_{n} .
$$

(b) There is a $k$ such that

$$
\liminf _{n \rightarrow \infty} \sigma_{k+1}^{(n)}>0 .
$$

This theorem suggests introducing the $\alpha$-number $\alpha\left(\left(A_{n}\right)\right)$ of a Fredholm sequence $\left(A_{n}\right)$ (corresponding to the kernel dimension of a Fredholm operator) as the smallest number $k$ for which (10) is true. Equivalently, $\alpha\left(\left(A_{n}\right)\right)$ is the smallest number for which there exists a sequence $\left(B_{n}\right) \in$ $\mathcal{F}$ as well as a sequence $\left(J_{n}\right) \in \mathcal{K}$ such that $B_{n} A_{n}^{*} A_{n}=I_{n}+J_{n}$ and $\lim \sup _{n \rightarrow \infty} \operatorname{dim} \operatorname{Im} J_{n}=\alpha\left(\left(A_{n}\right)\right)$. The index of a Fredholm sequence is the quantity

$$
\operatorname{ind}\left(\left(A_{n}\right)\right):=\alpha\left(\left(A_{n}\right)\right)-\alpha\left(\left(A_{n}^{*}\right)\right) .
$$

Observe that, in the case at hand, this index is always zero. This is a consequence of the fact that the entries of the sequences under consideration are finite-dimensional operators and, hence, the matrices $A_{n}^{*} A_{n}$ and $A_{n} A_{n}^{*}$ have the same eigenvalues even with respect to their multiplicity. So the most interesting quantity associated with a Fredholm sequence of matrices seems to be its $\alpha$-number. On the other hand, the vanishing of the index of $\left(A_{n}\right)$ has remarkable consequences as has been pointed out in [5].

Let us still emphasize that, in general, the singular values of a compact or a Fredholm sequence do not exhibit the behaviour one would expect from the knowledge about the behaviour of the singular values of a compact or a Fredholm operator. Indeed, if $K$ is a compact operator on a Hilbert space, then there are at most countably many singular values, and all non-zero singular values are isolated points. Similarly, if $A$ is a Fredholm operator on a Hilbert space, then its range is closed and $A$ is Moore-Penrose invertible. The latter property is satisfied if and only if either $A^{*} A$ is invertible or if 0 is an isolated point in the spectrum of $A^{*} A$. Thus, one might expect that the non-zero singular values of the coset $\left(K_{n}\right)+\mathcal{G}$ of a compact sequence $\left(K_{n}\right)$ or the singular value 0 of the coset $\left(A_{n}\right)+\mathcal{G}$ of a non-stable Fredholm sequence $\left(A_{n}\right)$ are isolated. But this is not true as the following simple example shows.

EXAMPLE. Let $\left(a_{n}\right)$ be an enumeration of the rational numbers in $[0,1]$, and set

$$
\begin{aligned}
K_{n} & :=a_{n} P_{n} P_{1} P_{n}=\operatorname{diag}\left(a_{n}, 0, \ldots, 0\right), \\
A_{n} & :=K_{n}+P_{n}\left(I-P_{1}\right) P_{n}=\operatorname{diag}\left(a_{n}, 1, \ldots, 1\right) .
\end{aligned}
$$


The sequence $\left(K_{n}\right)$ consists of rank $\leq 1$ matrices; hence $\left(K_{n}\right)$ is a compact and $\left(A_{n}\right)$ is a Fredholm sequence. But both the largest singular values of the matrices $K_{n}$ and the smallest singular values of the matrices $A_{n}$ lie dense in $[0,1]$. Thus, the spectrum of both cosets $\left(K_{n}^{*} K_{n}\right)+\mathcal{G}$ and $\left(A_{n}^{*} A_{n}\right)+\mathcal{G}$ is $[0,1]$.

We will see in the following sections that compact and Fredholm sequences in fractal subalgebras show a behaviour of the singular values which is in perfect accordance with the behaviour of the singular values of compact and Fredholm operators.

3. Compact sequences in fractal algebras. In this section, we consider compact sequences $\left(K_{n}\right)$ in fractal algebras. In particular, the announced behaviour of the singular values of the matrices $K_{n}$ will be established. We start by recalling the definition of a fractal algebra and of some properties of fractal algebras from $[7,5]$.

Given a strongly increasing sequence $\eta: \mathbb{N} \rightarrow \mathbb{N}$, let $\mathcal{F}_{\eta}$ be the $C^{*}$-algebra of all bounded sequences $\left(A_{n}\right)$ with $A_{n} \in \mathbb{C}^{\eta(n) \times \eta(n)}$, and write $\mathcal{G}_{\eta}$ for the ideal of all sequences $\left(A_{n}\right) \in \mathcal{F}_{\eta}$ which tend to zero in norm. Further, let $R_{\eta}$ stand for the restriction mapping $R_{\eta}: \mathcal{F} \rightarrow \mathcal{F}_{\eta},\left(a_{n}\right) \mapsto\left(a_{\eta(n)}\right)$. This mapping is a *-homomorphism from $\mathcal{F}$ onto $\mathcal{F}_{\eta}$ which moreover maps $\mathcal{G}$ onto $\mathcal{G}_{\eta}$. Further, given a $C^{*}$-subalgebra $\mathcal{A}$ of $\mathcal{F}$, let $\mathcal{A}_{\eta}$ denote the image of $\mathcal{A}$ under $R_{\eta}$ which is a $C^{*}$-algebra again.

Definition 3.1. Let $\mathcal{A}$ be a $C^{*}$-subalgebra of the algebra $\mathcal{F}$.

(a) $\mathrm{A}^{*}$-homomorphism $W: \mathcal{A} \rightarrow \mathcal{B}$ of $\mathcal{A}$ into a $C^{*}$-algebra $\mathcal{B}$ is fractal if, for every strongly increasing sequence $\eta$, there is a ${ }^{*}$-homomorphism $W_{\eta}: \mathcal{A}_{\eta} \rightarrow \mathcal{B}$ such that $W=W_{\eta} R_{\eta}$.

(b) The algebra $\mathcal{A}$ is fractal if the canonical homomorphism $\pi: \mathcal{A} \rightarrow$ $\mathcal{A} /(\mathcal{A} \cap \mathcal{G}),\left(A_{n}\right) \mapsto\left(A_{n}\right)+(\mathcal{A} \cap \mathcal{G})$, is fractal.

(c) A sequence $\left(A_{n}\right) \in \mathcal{F}$ is fractal if the smallest $C^{*}$-subalgebra of $\mathcal{F}$ which contains $\left(A_{n}\right)$ is fractal.

Thus, given a subsequence $\left(A_{\eta(n)}\right)$ of a sequence $\left(A_{n}\right)$ which belongs to a fractal algebra $\mathcal{A}$, it is possible to reconstruct the original sequence $\left(A_{n}\right)$ from this subsequence modulo sequences in $\mathcal{A} \cap \mathcal{G}$. This assumption is very natural for sequences arising from discretization procedures, and it is indeed satisfied for a lot of concrete approximation sequences; see e.g. [6]. On the other hand, the algebra $\mathcal{F}$ of all bounded sequences fails to be fractal.

Here are a few properties of fractal sequences proved in [5].

TheOREM 3.2. A $C^{*}$-subalgebra $\mathcal{A}$ of $\mathcal{F}$ is fractal if and only if, for every $\left(A_{n}\right) \in \mathcal{A}$ and every strongly increasing sequence $\eta$,

$$
R_{\eta}\left(A_{n}\right) \in \mathcal{G}_{\eta} \Rightarrow\left(A_{n}\right) \in \mathcal{A} \cap \mathcal{G} .
$$


THEOREM 3.3. A sequence $\left(A_{n}\right) \in \mathcal{F}$ of self-adjoint matrices is fractal if and only if $\liminf \sigma\left(A_{n}\right)=\lim \sup \sigma\left(A_{n}\right)$ where $\sigma\left(A_{n}\right)$ stands for the spectrum of $A_{n}$.

If the limes superior $\lim \sup M_{n}$ and the limes inferior $\lim \inf M_{n}$ of a sequence $\left(M_{n}\right)$ of compact subsets of the complex plane coincide, then the sequence $\left(M_{n}\right)$ converges with respect to the Hausdorff distance, and we write $\lim M_{n}$ for the limit in the Hausdorff sense.

Proposition 3.4. If $\left(A_{n}\right) \in \mathcal{F}$ is a fractal sequence, then $\lim \left\|A_{n}\right\|$ exists and it is equal to the norm of the coset $\left(A_{n}\right)+\mathcal{G}$ in the quotient algebra $\mathcal{F} / \mathcal{G}$.

3.1. Singular values of fractal compact sequences. Let $\left(K_{n}\right) \in \mathcal{F}$ be a fractal sequence (not necessarily compact for the moment), and let $\Sigma_{1}^{(n)} \geq$ $\ldots \geq \Sigma_{n}^{(n)} \geq 0$ denote the singular values of $K_{n}$. Further we write $\sigma_{2}(A)$ for the set of singular values of an operator $A$.

LEMma 3.5. The sequence $\left(\Sigma_{1}^{(n)}\right)$ of the largest singular values of $K_{n}$ converges.

Proof. The assertion is an immediate consequence of Proposition 3.4 and of the identity $\Sigma_{1}^{(n)}=\left\|K_{n}\right\|$. Another short proof based on Theorem 3.3 runs as follows. Assume that $\left(\Sigma_{1}^{(n)}\right)$ has two limiting points, say $\alpha$ and $\beta$ with $\alpha>\beta$. Then $\beta$ is the limit of a subsequence $\left(\Sigma_{1}^{\left(n_{r}\right)}\right)_{r \geq 1}$. The point $\alpha$ cannot belong to $\limsup _{r \rightarrow \infty} \sigma_{2}\left(K_{n_{r}}\right)$ (the $\Sigma_{1}^{\left(n_{r}\right)}$ are the largest singular values of $K_{n_{r}}$, and they converge to $\beta$ which is less than $\alpha$ ). Hence,

$$
\alpha \in \lim \sup \sigma_{2}\left(K_{n}\right) \backslash \liminf \sigma_{2}\left(K_{n}\right),
$$

in contradiction to Theorem 3.3.

Observe that the sequence $\left(\Sigma_{2}^{(n)}\right)$ need not converge even if $\left(K_{n}\right)$ is a fractal sequence, as shown by the example

$$
K_{n}:= \begin{cases}\operatorname{diag}(1,0,0, \ldots, 0) & \text { if } n \text { is even, } \\ \operatorname{diag}(1,1,0, \ldots, 0) & \text { if } n \text { is odd. }\end{cases}
$$

But, as in the second proof of Lemma 3.5 , one can easily verify that $\left(\Sigma_{2}^{(n)}\right)$ can have at most two limiting points, at most one of which can be different from $\lim \Sigma_{1}^{(n)}$. More generally, the following is true, where we write $\Pi_{k}$ for the set of all partial limits of the sequence $\left(\Sigma_{k}^{(n)}\right)_{n \geq k}$.

Proposition 3.6. If $\left(K_{n}\right) \in \mathcal{F}$ is fractal, then $\Pi_{k+1} \backslash \Pi_{k}$ contains at most one element. 
Proof. We start by showing the identity

$$
\Pi_{1} \cup \ldots \cup \Pi_{k}=\limsup _{n \rightarrow \infty}\left\{\Sigma_{1}^{(n)}, \ldots, \Sigma_{k}^{(n)}\right\} \quad \text { for every } k .
$$

The inclusion $\subseteq$ is evident. Conversely, if $\lambda \in \limsup _{n \rightarrow \infty}\left\{\Sigma_{1}^{(n)}, \ldots, \Sigma_{k}^{(n)}\right\}$, then there are a subsequence $\left(n_{r}\right)$ of $\mathbb{N}$ and numbers $k_{r}$ in $\{1, \ldots, k\}$ such that

$$
\lambda=\lim _{r \rightarrow \infty} \Sigma_{k_{r}}^{\left(n_{r}\right)} .
$$

Since the $k_{r}$ can take only finitely many values, there is a $k_{0}$ between 1 and $k$ such that

$$
\lambda=\lim _{r \rightarrow \infty} \Sigma_{k_{0}}^{\left(n_{r}\right)} .
$$

Hence, $\lambda \in \Pi_{k_{0}}$, which shows (11).

Now we proceed as in the second proof of Lemma 3.5. Assume that the sequence $\left(\Sigma_{k+1}^{(n)}\right)_{n}$ has two limiting points $\alpha$ and $\beta$ with $\alpha>\beta$, both not belonging to $\Pi_{k}$. Choose a subsequence $\left(\Sigma_{k+1}^{\left(n_{r}\right)}\right)_{r \geq 1}$ which converges to $\beta$ as $r \rightarrow \infty$. Then $\alpha$ cannot belong to $\lim \sup _{r \rightarrow \infty} \sigma_{2}\left(K_{n_{r}}\right)$. Indeed, since $\alpha \notin \Pi_{k}$ by assumption, $\alpha$ cannot belong to

$$
\Pi_{1} \cup \ldots \cup \Pi_{k}=\limsup _{n \rightarrow \infty}\left\{\Sigma_{1}^{(n)}, \ldots, \Sigma_{k}^{(n)}\right\}
$$

due to monotony reasons. But the $(k+1)$ th singular values of $K_{n_{r}}$ converge to $\beta<\alpha$. Hence,

$$
\alpha \in \lim \sup \sigma_{2}\left(K_{n}\right) \backslash \liminf \sigma_{2}\left(K_{n}\right),
$$

in contradiction to Theorem 3.3.

Corollary 3.7. If $\left(K_{n}\right) \in \mathcal{F}$ is fractal, then $\limsup _{n \rightarrow \infty}\left\{\Sigma_{1}^{(n)}, \ldots\right.$ $\left.\ldots, \Sigma_{k}^{(n)}\right\}$ contains at most $k$ elements.

Now we can show that, with respect to the singular values, fractal compact sequences behave as compact operators on Hilbert space.

Corollary 3.8. Let $\left(K_{n}\right) \in \mathcal{K}$ be a fractal sequence and $\varepsilon>0$. Then there are only finitely many points in $\lim \sigma_{2}\left(K_{n}\right)$ with absolute value larger than $\varepsilon$.

Proof. By Theorem 2.1, $\lim _{k \rightarrow \infty} \sup _{n \geq k} \Sigma_{k}^{(n)}=0$. Thus, there is a $k_{0}$ such that $\sup _{n \geq k_{0}} \Sigma_{k_{0}}^{(n)} \leq \varepsilon$, whence

$$
\limsup _{n \rightarrow \infty}\left\{\Sigma_{k_{0}}^{(n)}, \ldots, \Sigma_{n}^{(n)}\right\} \subseteq[0, \varepsilon] .
$$

Since

$$
\lim _{n \rightarrow \infty} \sigma_{2}\left(K_{n}\right)=\limsup _{n \rightarrow \infty}\left\{\Sigma_{1}^{(n)}, \ldots, \Sigma_{k_{0}-1}^{(n)}\right\} \cup \limsup _{n \rightarrow \infty}\left\{\Sigma_{k_{0}}^{(n)}, \ldots, \Sigma_{n}^{(n)}\right\},
$$


it is obvious that the points in $\lim \sigma_{2}\left(K_{n}\right)$ with absolute value larger than $\varepsilon$ lie in

$$
\limsup _{n \rightarrow \infty}\left\{\Sigma_{1}^{(n)}, \ldots, \Sigma_{k_{0}-1}^{(n)}\right\} .
$$

This set is finite by Corollary 3.7 .

Corollary 3.9. Let $\left(K_{n}\right) \in \mathcal{K}$ be fractal. Then $0 \in \lim \sigma_{2}\left(K_{n}\right)$, the set $\lim \sigma_{2}\left(K_{n}\right)$ is at most countable, and 0 is its only limiting point.

3.2. The ideal $(\mathcal{A} \cap \mathcal{K}) / \mathcal{G}$. Throughout what follows let $\mathcal{A}$ be a unital fractal $C^{*}$-subalgebra of $\mathcal{F}$ which contains the ideal $\mathcal{G}$. We will now apply the results of the preceding section to analyse the ideal $\mathcal{A} \cap \mathcal{K}$ of compact sequences in $\mathcal{A}$.

Generators of $(\mathcal{A} \cap \mathcal{K}) / \mathcal{G}$. Our first goal is to show that the ideal $\mathcal{A} \cap \mathcal{K}$ is generated by its projections. Later on we will see that it is even generated by its minimal projections.

THEOREM 3.10. The ideal $\mathcal{A} \cap \mathcal{K}$ is generated (as a $C^{*}$-algebra or as an ideal of $\mathcal{A})$ by its projections, i.e. by the sequences $\left(Q_{n}\right) \in \mathcal{A} \cap \mathcal{K}$ where each matrix $Q_{n}$ is a self-adjoint idempotent.

Proof. Every sequence in $\mathcal{A} \cap \mathcal{K}$ is a linear combination of four nonnegative sequences in $\mathcal{A} \cap \mathcal{K}$, and every non-negative sequence in $\mathcal{A} \cap \mathcal{K}$ is of the form $\left(K_{n}^{*} K_{n}\right)$ with a sequence $\left(K_{n}\right) \in \mathcal{A} \cap \mathcal{K}$. The assertion will follow once we show that, for every sequence $\left(K_{n}\right) \in \mathcal{A} \cap \mathcal{K}$, the sequence $\left(K_{n}^{*} K_{n}\right)$ can be approximated in the norm of $\mathcal{F}$ by linear combinations of projections in $\mathcal{A} \cap \mathcal{K}$.

By Corollary 3.9, there are (finitely or countably many) numbers $\lambda_{1}>$ $\lambda_{2}>\ldots>0$ such that

$$
\sigma_{\mathcal{F}}\left(\left(K_{n}^{*} K_{n}\right)\right)=\left\{\lambda_{1}, \lambda_{2}, \ldots\right\} \cup\{0\}=: \Lambda .
$$

The smallest $C^{*}$-subalgebra $\mathcal{K}^{\prime}$ of $\mathcal{F}$ which contains the sequences $\left(K_{n}^{*} K_{n}\right)$ and $\left(I_{n}\right)$ is *-isometric to $C(\Lambda)$, and the Gelfand transform maps $\left(K_{n}^{*} K_{n}\right)$ to the identity mapping on $\Lambda$. For every $\lambda_{k}$, let $\mathcal{P}_{k}$ be the sequence in $\mathcal{K}^{\prime}=C(\Lambda)$ whose Gelfand transform is 1 at $\lambda_{k}$ and 0 on $\Lambda \backslash\left\{\lambda_{k}\right\}$ (this function is obviously continuous on $\Lambda$ ). Each $\mathcal{P}_{k}$ is a projection in $\mathcal{K}^{\prime} \subseteq \mathcal{A} \cap \mathcal{K}$, and

$$
\left\|\left(K_{n}^{*} K_{n}\right)-\sum_{i=1}^{r} \lambda_{i} \mathcal{P}_{i}\right\|_{\mathcal{F}}= \begin{cases}\lambda_{k+1} & \text { if } \Lambda \text { has at least } k+2 \text { elements } \\ 0 & \text { if } \Lambda \text { has at most } k+1 \text { elements. }\end{cases}
$$

Since $\lambda_{k} \rightarrow 0$ as $k \rightarrow \infty$, this implies the assertion.

Due to Theorem 3.3, one of the following possibilities is satisfied for every fractal sequence $\left(Q_{n}\right)$ of projections: either $\sigma\left(Q_{n}\right)=\{1\}$, or $\sigma\left(Q_{n}\right)=\{0\}$, or $\sigma\left(Q_{n}\right)=\{0,1\}$, for all sufficiently large $n$. In the first case we have $Q_{n}=I_{n}$ for all sufficiently large $n$, and the sequence $\left(Q_{n}\right)$ cannot belong 
to $\mathcal{K}$ (Corollary 2.2). In the second case, $Q_{n}=0$ for all large $n$, and the sequence $\left(Q_{n}\right)$ lies in $\mathcal{G}$.

We call a sequence $\left(Q_{n}\right) \in \mathcal{F}$ of projections essential if $1 \in \sigma\left(Q_{n}\right)$ for all $n$.

Proposition 3.11. The ideal $\mathcal{A} \cap \mathcal{K}$ is generated (as an ideal in $\mathcal{A}$ ) by its essential projections.

Proof. For a moment, let $\mathcal{K}^{\prime}$ stand for the smallest closed ideal of $\mathcal{A}$ which contains all essential projections from $\mathcal{A} \cap \mathcal{K}$. Evidently, $\mathcal{K}^{\prime} \subseteq \mathcal{A} \cap \mathcal{K}$. For the reverse inclusion, we have to show that $\mathcal{K}^{\prime}$ contains all projections in $\mathcal{A} \cap \mathcal{K}$ (Theorem 3.10). For this goal, we first check that

$$
\mathcal{G} \subseteq \mathcal{K}^{\prime}
$$

Let $r \in \mathbb{N}$ and $\left(Q_{n}\right) \in \mathcal{A} \cap \mathcal{K}$ be an essential sequence of projections. The sequence $\left(0, \ldots, 0, I_{r}, 0, \ldots\right)$ with $I_{r}$ standing at the $r$ th place lies in $\mathcal{A}$; hence, the sequence $\left(0, \ldots, 0, Q_{r}, 0, \ldots\right)$ belongs to $\mathcal{K}^{\prime}$. Since $Q_{r} \neq 0$, the smallest closed ideal of $\mathbb{C}^{r \times r}$ which contains $Q_{r}$ is equal to $\mathbb{C}^{r \times r}$, which shows that $\mathcal{K}^{\prime}$ contains all sequences $\left(0, \ldots, 0, G_{r}, 0, \ldots\right)$ with $G_{r} \in \mathbb{C}^{r \times r}$. Consequently, all sequences $\left(G_{1}, G_{2}, \ldots, G_{k}, 0,0, \ldots\right)$ belong to $\mathcal{K}^{\prime}$. Since $\mathcal{G}$ is the closure of the set of all sequences of this kind, (12) holds.

Let now $\left(R_{n}\right) \in \mathcal{A} \cap \mathcal{K}$ be a projection. By the above classification, either $\sigma\left(R_{n}\right)=\{0\}$ or $\sigma\left(R_{n}\right)=\{0,1\}$, for all sufficiently large $n$. In the first case, $\left(R_{n}\right) \in \mathcal{G}$, whence $\left(R_{n}\right) \in \mathcal{K}^{\prime}$ due to $(12)$. In the second case, at most finitely many of the matrices $R_{n}$ are zero. We replace them by $I_{n}$ and get a new sequence $\left(R_{n}^{\prime}\right)$. This sequence is an essential projection and, hence, is in $\mathcal{K}^{\prime}$ by definition, and the difference $\left(R_{n}^{\prime}\right)-\left(R_{n}\right)$ is in $\mathcal{G}$ by construction and in $\mathcal{K}^{\prime}$ due to (12).

Later, we will need the following simple consequence of the preceding proposition.

Corollary 3.12. If $q \in(\mathcal{A} \cap \mathcal{K}) / \mathcal{G}$ is a non-zero projection, then there is an essential projection $\left(Q_{n}\right) \in \mathcal{A} \cap \mathcal{K}$ such that $\left(Q_{n}\right)+\mathcal{G}=q$.

We also say that $q$ can be lifted to an essential projection. For the proof note that every projection $q \in \mathcal{F} / \mathcal{G}$ can be lifted to a projection $\left(R_{n}\right) \in \mathcal{F}$ (see, e.g., [7]). Since $q \neq 0$ and $q \in \mathcal{K} / \mathcal{G}$, we have $\left(R_{n}\right) \notin \mathcal{G}$ and $\left(R_{n}\right) \notin$ $\left(P_{n}\right)+\mathcal{G}$. Thus, $\sigma\left(R_{n}\right)=\{0,1\}$ for all sufficiently large $n$, and $\left(R_{n}\right)$ can be made an essential projection by adding a sequence in $\mathcal{G}$ as in the proof of Proposition 3.11.

An order relation for projections. In what follows we will have to compare projections in $(\mathcal{A} \cap \mathcal{K}) / \mathcal{G}$. The needed definitions will be given in a more general context. 
Let $\mathfrak{B}$ be a $C^{*}$-algebra, and let $q, r$ be projections (i.e. self-adjoint idempotents) in $\mathfrak{B}$. We say that $r \preceq q$ if $r q r=r$. If $r \preceq q$ and $r \neq q$, then we write $r \prec q$. Obviously, $0 \preceq q$, and if $\mathfrak{B}$ has an identity element $e$, then $q \preceq e$ for every projection $q$.

If $r q r=r$ for projections $q$, $r$, then $(i(q r-r q))^{3}=0$ as one easily verifies. Since $i(q r-r q)$ is self-adjoint, this observation implies that $q r=r q$ whenever $r q r=r$. With this commutativity, it is trivial to check that the relation $\preceq$ is reflexive, symmetric and transitive, i.e. an order relation.

Lemma 3.13. Let $q, r$ be projections in a $C^{*}$-algebra $\mathfrak{B}$ such that $r \preceq q$. Then $q-r$ is also a projection, and $q-r \preceq q$.

Proof. We have already remarked that $r \preceq q$ implies $r q r=r q=q r=r$. Hence,

$$
(q-r)^{2}=q^{2}-q r-r q+r^{2}=q-r
$$

and

$$
(q-r) q(q-r)=q^{3}-q^{2} r-r q^{2}+r q r=q-q r-r q+r=q-r .
$$

The first identity shows that $q-r$ is a projection, the second that $q-r \preceq q$.

We call a projection $p \in \mathfrak{B}$ minimal if, for every projection $q \in \mathfrak{B}$ with $q \preceq p, q=0$ or $q=p$. Clearly, 0 is a minimal projection, the trivial minimal projection. The quotient $l^{\infty} / c_{0}$ is an example of a $C^{*}$-algebra without nontrivial minimal projections. Further, an element $r \in \mathfrak{B}$ is said to be of rank one if, for every $b \in \mathfrak{B}$, there is a $\beta \in \mathbb{C}$ such that $r b r=\beta r$.

Proposition 3.14. Let $\mathfrak{B}$ be a $C^{*}$-algebra. Then every projection of rank one is minimal.

Proof. Let $p \in \mathfrak{B}$ be a projection of rank one. If $p=0$, then there is nothing to prove. So let $p \neq 0$, and let $q \in \mathfrak{B}$ be a projection with $q \preceq p$, i.e. $q p q=q$. Since $p$ is of rank one, there is a $\mu \in \mathbb{C}$ such that $p q p=\mu p$. So one has

$$
q=q^{2}=(q p q)^{2}=q(p q p) q=\mu q p q=\mu q,
$$

whence $q=0$ or $\mu=1$. If $\mu=1$, then $p q p=p$, i.e. $p \preceq q$. Due to the symmetry of $\preceq$, this implies $p=q$. Thus, in any case, $q=0$ or $q=p$, i.e. $p$ is minimal.

The converse of Proposition 3.14 is not true. If, for instance, $\mathfrak{B}=$ $C([0,1] \cup[2,3])$, then the function which is 1 on $[0,1]$ and 0 on $[2,3]$ is a minimal projection in $\mathfrak{B}$ which is not of rank one. We will see now that for the algebras we shall be concerned with, the classes of minimal projections and of rank one projections coincide.

Theorem 3.15. Let $\mathcal{A}$ be a unital fractal $C^{*}$-subalgebra of $\mathcal{F}$ which contains the ideal $\mathcal{G}$. Then every minimal projection in $(\mathcal{A} \cap \mathcal{K}) / \mathcal{G}$ is a rank one projection in $\mathcal{A} / \mathcal{G}$. 
Proof. Let $q$ be a minimal projection in $(\mathcal{A} \cap \mathcal{K}) / \mathcal{G}$. If $q=0$, then $q$ is of rank one. So let $q \neq 0$ and $a \in \mathcal{A} / \mathcal{G}$. We have to show that there is an $\alpha \in \mathbb{C}$ such that $q a q=\alpha q$. Since $a$ can be written as a linear combination of four non-negative elements, it is sufficient to verify that, for every $b \in \mathcal{A} / \mathcal{G}$, there is a $\beta \in \mathbb{C}$ such that

$$
q b^{*} b q=\beta q .
$$

Let $\mathcal{C}$ stand for the smallest $C^{*}$-subalgebra of $\mathcal{A} / \mathcal{G}$ which contains the elements $q$ and $q b^{*} b q$. This algebra is commutative, has $q$ as its identity element, and its maximal ideal space is homeomorphic to $\sigma_{\mathcal{C}}\left(q b^{*} b q\right)$.

Choose sequences $\left(Q_{n}\right) \in \mathcal{A} \cap \mathcal{K}$ and $\left(B_{n}\right) \in \mathcal{A}$ with $\left(Q_{n}\right)+\mathcal{G}=q$ and $\left(B_{n}\right)+\mathcal{G}=b$. From Corollary 3.9 we infer that

$$
\sigma_{\mathcal{A}}\left(\left(Q_{n} B_{n}^{*} B_{n} Q_{n}\right)_{n \geq 1}\right)=\{0\} \cup\left\{\lambda_{1}, \lambda_{2}, \ldots\right\}=: \Lambda
$$

with numbers $\lambda_{1}>\lambda_{2}>\ldots>0$ having 0 as their only possible accumulation point. Hence, $\sigma_{\mathcal{A} / \mathcal{G}}\left(q b^{*} b q\right) \subseteq \Lambda$. We claim that

$$
\sigma_{\mathcal{C}}\left(q b^{*} b q\right) \subseteq \sigma_{\mathcal{A} / \mathcal{G}}\left(q b^{*} b q\right)
$$

which clearly implies that

$$
\sigma_{\mathcal{C}}\left(q b^{*} b q\right) \subseteq \Lambda .
$$

To prove (14), let $\lambda \in \mathbb{C} \backslash \sigma_{\mathcal{A} / \mathcal{G}}\left(q b^{*} b q\right)$. Then there is a $c$ in $\mathcal{A} / \mathcal{G}$ such that $\left(q b^{*} b q-\lambda e\right) c=e$. Multiplying this identity by $q$ we get $\left(q b^{*} b q-\lambda q\right) q c q=q$, i.e. $q b^{*} b q-\lambda q$ is invertible in the $C^{*}$-algebra $q(\mathcal{A} / \mathcal{G}) q$ with identity $q$. But then, by the inverse closedness of $C^{*}$-algebras, $q b^{*} b q-\lambda q$ is also invertible in the $C^{*}$-subalgebra $\mathcal{C}$ of $q(\mathcal{A} / \mathcal{G}) q$. Hence, $\lambda \in \mathbb{C} \backslash \sigma_{\mathcal{C}}\left(q b^{*} b q\right)$, which yields (14).

Now assume that $\sigma_{\mathcal{C}}\left(q b^{*} b q\right)$ contains at least two points. Then at least one of them, say $\lambda_{0}$, is not 0 . We consider the element $p \in \mathcal{C}$ whose Gelfand transform satisfies

$$
p\left(\lambda_{0}\right)=1, \quad p(\lambda)=0 \quad \text { for all } \lambda \in \sigma_{\mathcal{C}}\left(q b^{*} b q\right) \backslash\left\{\lambda_{0}\right\}
$$

(this function is continuous on $\sigma_{\mathcal{C}}\left(q b^{*} b q\right)$ due to (15) and the choice of $\lambda_{0}$ ). The element $p$ is a projection in $\mathcal{C} \subseteq(\mathcal{A} \cap \mathcal{K}) / \mathcal{G}$, and $p \preceq q$ since $q(\lambda)=1$ for all $\lambda \in \sigma_{\mathcal{C}}\left(q b^{*} b q\right)$. But, evidently, $p$ is neither 0 (since $p\left(\lambda_{0}\right)=1$ ) nor $q$ (since $\sigma_{\mathcal{C}}\left(q b^{*} b q\right)$ contains at least two points by assumption). This contradicts the minimality of $q$. Hence, the maximal ideal space $\sigma_{\mathcal{C}}\left(q b^{*} b q\right)$ of $\mathcal{C}$ consists of exactly one point. But then every element of $\mathcal{C}$ is a multiple of the identity element whence there exists a $\beta \in \mathbb{C}$ such that (13) holds.

It is also clear that $\beta \geq 0$ : Since $\sigma_{\mathcal{A} / \mathcal{G}}(q)=\{0,1\}$, one has $\sigma_{\mathcal{A} / \mathcal{G}}(\beta q)=$ $\{0, \beta\}$ and, consequently,

$$
\sigma_{\mathcal{A} / \mathcal{G}}\left(q b^{*} b q\right)=\{0, \beta\} .
$$

Because $q b^{*} b q \geq 0$, this implies $\beta \geq 0$. 
Minimal generators. The next goal is to show that the ideal $(\mathcal{A} \cap \mathcal{K}) / \mathcal{G}$ not only contains minimal projections, but is even generated by its minimal projections. We start by considering the consequences of the relation $r \preceq q$ between two projections $q, r \in(\mathcal{A} \cap \mathcal{K}) / \mathcal{G}$ for the representatives of these cosets.

Theorem 3.16. Let $\mathcal{A}$ be as in the preceding theorem, and let $q, r \in$ $(\mathcal{A} \cap \mathcal{K}) / \mathcal{G}$ be projections with $r \preceq q$. Let further $q=\left(Q_{n}\right)+\mathcal{G}$ and $r=$ $\left(R_{n}\right)+\mathcal{G}$ with projections $\left(Q_{n}\right),\left(R_{n}\right) \in \mathcal{A} \cap \mathcal{K}$ (these projections exist and can be chosen as essential projections due to Corollary 3.12). If $r \neq q$, then

$$
\operatorname{dim} \operatorname{Im} R_{n}<\operatorname{dim} \operatorname{Im} Q_{n}
$$

for all $n \in \mathbb{N}$ with at most finitely many exceptions.

In the proof, we will make use of the following simple observation: Let $P$ be an orthogonal projection on a Hilbert space $H$, and let $A \in L(H)$ be an operator such that $\|P-A\|<1$. Then $\|P-P A P\|=\|P(P-A) P\| \leq$ $\|P-A\|<1$. Hence, by Neumann series, the operator

$$
P A P=P-(P-P A P): \operatorname{Im} P \rightarrow \operatorname{Im} P
$$

is invertible, whence

$$
\operatorname{dim} \operatorname{Im} P A P=\operatorname{dim} \operatorname{Im} P .
$$

Proof of Theorem 3.16. Since $r q r=r$, one has $r=r q=q r=r q r$. Consequently,

$$
\begin{aligned}
& \left\|R_{n}-Q_{n} R_{n} Q_{n}\right\| \rightarrow 0, \\
& \left\|R_{n}-R_{n} Q_{n}\right\| \rightarrow 0 \text { and }\left\|R_{n}-Q_{n} R_{n}\right\| \rightarrow 0 \quad \text { as } n \rightarrow \infty .
\end{aligned}
$$

Due to (17),

$$
\left\|R_{n}-Q_{n} R_{n} Q_{n}\right\|<1 \quad \text { for all sufficiently large } n \text {. }
$$

Thus, by (16),

$$
\operatorname{dim} \operatorname{Im} R_{n}=\operatorname{dim} \operatorname{Im} R_{n} Q_{n} R_{n} \leq \operatorname{dim} \operatorname{Im} Q_{n}
$$

for all sufficiently large $n$. Suppose that $\operatorname{dim} \operatorname{Im} R_{n}=\operatorname{dim} \operatorname{Im} Q_{n}$ for all $n$ in an infinite subset $\eta$ of $\mathbb{N}$. Then, for every $n \in \eta$, there is a unitary matrix $U_{n}: \mathbb{C}^{n} \rightarrow \mathbb{C}^{n}$ such that $R_{n}=U_{n} Q_{n} U_{n}^{*}$. (Indeed, choose orthonormal bases $\left\{e_{1}, \ldots, e_{k}\right\}$ and $\left\{f_{1}, \ldots, f_{k}\right\}$ of $\operatorname{Im} Q_{n}$ and $\operatorname{Im} R_{n}$, complete these bases to orthonormal bases $\left\{e_{1}, \ldots, e_{n}\right\}$ and $\left\{f_{1}, \ldots, f_{n}\right\}$ of $\mathbb{C}^{n}$, and set $U_{n} e_{r}:=f_{r}$ for $1 \leq r \leq n$.) Thus, for every $n \in \eta$,

$$
\begin{aligned}
\left\|R_{n}-R_{n} Q_{n}\right\| & =\left\|U_{n} Q_{n} U_{n}^{*}-U_{n} Q_{n} U_{n}^{*} Q_{n}\right\|=\left\|Q_{n}-Q_{n} U_{n}^{*} Q_{n} U_{n}\right\| \\
& =\left\|Q_{n}-Q_{n} R_{n}\right\| .
\end{aligned}
$$

Together with the first assertion of (18), this shows that $\left(Q_{n}-Q_{n} R_{n}\right)_{n \in \eta}$ $\in \mathcal{G}_{\eta}$ and, together with the second assertion of (18), we even deduce that 
$\left(R_{n}-Q_{n}\right)_{n \in \eta} \in \mathcal{G}_{\eta}$. Via Theorem 3.2, the fractality of $\mathcal{A}$ implies that $\left(R_{n}-Q_{n}\right)_{n \in \mathbb{N}} \in \mathcal{G}$, i.e. $q=r$ contrary to the hypotheses.

Corollary 3.17. Let $\mathcal{A}$ be as in the preceding theorem. Then every chain $q_{0} \succ q_{1} \succ q_{2} \succ \ldots$ of projections $q_{i} \in(\mathcal{A} \cap \mathcal{K}) / \mathcal{G}$ is finite.

Proof. Let $\left(Q_{n}\right) \in \mathcal{A} \cap \mathcal{K}$ be a projection such that $\left(Q_{n}\right)+\mathcal{G}=q_{0}$. By the definition of $\mathcal{K}$, there is a sequence $\left(K_{n}\right) \in \mathcal{F}$ with $l:=\sup _{n} \operatorname{dim} \operatorname{Im} R_{n}<\infty$ such that $\left\|Q_{n}-R_{n}\right\|<1$ for all $n$. Then, by (16),

$$
\operatorname{dim} \operatorname{Im} Q_{n}=\operatorname{dim} \operatorname{Im} Q_{n} R_{n} Q_{n} \leq \operatorname{dim} \operatorname{Im} R_{n} \leq l
$$

for all $n$. In particular, $l_{0}:=\sup _{n} \operatorname{dim} \operatorname{Im} Q_{n}<\infty$. For $j \geq 1$, let $\left(Q_{n}^{(j)}\right) \in$ $\mathcal{A} \cap \mathcal{K}$ be a projection such that $\left(Q_{n}^{(j)}\right)+\mathcal{G}=q_{j}$. Repeated application of Theorem 3.16 shows that

$$
\begin{array}{ll}
\operatorname{dim} \operatorname{Im} Q_{n}^{(1)} \leq l_{0}-1 & \text { for almost all } n, \\
\operatorname{dim} \operatorname{Im} Q_{n}^{(2)} \leq l_{0}-2 & \text { for almost all } n, \ldots
\end{array}
$$

where for almost all $n$ means for all $n$ with finitely many exceptions. Thus, after at most $l_{0}$ steps, $\operatorname{dim} \operatorname{Im} Q_{n}^{(1)}=0$ for almost all $n$, i.e. $q_{l_{0}}=0$.

Corollary 3.18. For every non-trivial projection $q \in(\mathcal{A} \cap \mathcal{K}) / \mathcal{G}$, there is a non-trivial minimal projection $r \in(\mathcal{A} \cap \mathcal{K}) / \mathcal{G}$ such that $r \preceq q$.

Proof. If $q$ is not minimal, then there is a projection $q_{1} \in(\mathcal{A} \cap \mathcal{K}) / \mathcal{G}$ with $q_{1} \prec q$. If also $q_{1}$ is not minimal, then there is a projection $q_{2} \in(\mathcal{A} \cap \mathcal{K}) / \mathcal{G}$ such that $q_{2} \prec q_{1}$, etc. By Corollary 3.17, the chain $q \succ q_{1} \succ q_{2} \succ \ldots$ has a smallest non-trivial entry which is a minimal projection.

Corollary 3.19. Every projection $q \in(\mathcal{A} \cap \mathcal{K}) / \mathcal{G}$ is a finite sum of minimal projections in $(\mathcal{A} \cap \mathcal{K}) / \mathcal{G}$.

Proof. If $q=0$ then $q$ is minimal. So let $q \in(\mathcal{A} \cap \mathcal{K}) / \mathcal{G}$ be a non-trivial projection. If $q$ is minimal, then again there is nothing to prove. If $q$ is not minimal, then there exists a non-trivial minimal projection $p_{1} \prec q$ by Corollary 3.18. The element $q-p_{1}$ is a projection by Lemma 3.13. If $q-p_{1}$ is minimal, then $q=p_{1}+\left(q-p_{1}\right)$ is a sum of two minimal projections, and we are done. If $q-p_{1}$ is not minimal, then (again by Corollary 3.18) there is a non-trivial minimal projection $p_{2} \prec q-p_{1}$. If $q-p_{1}-p_{2}$ is minimal, then $q=p_{1}+p_{2}+\left(q-p_{1}-p_{2}\right)$ is a sum of three minimal projections. Otherwise, we proceed in this manner to find a non-trivial projection $p_{3}$ such that $p_{3} \prec q-p_{1}-p_{2}$. Since the chain

$$
q \succ q-p_{1} \succ q-p_{1}-p_{2} \succ \ldots
$$

is finite by Corollary 3.17, this process terminates, i.e. there is a non-trivial minimal projection $q-p_{1}-\ldots-p_{r}$. Then $q=p_{1}+\ldots+p_{r}+\left(q-p_{1}-\ldots-p_{r}\right)$ is the sum of $r+1$ non-trivial minimal projections. 
Combining this result with Theorem 3.10 we get:

TheOREm 3.20. Let $\mathcal{A}$ be a unital fractal $C^{*}$-subalgebra of $\mathcal{F}$ which contains the ideal $\mathcal{G}$. Then the ideal $(\mathcal{A} \cap \mathcal{K}) / \mathcal{G}$ is (as an ideal of $\mathcal{A} / \mathcal{G})$ generated by its non-trivial minimal projections.

Equivalently, $(\mathcal{A} \cap \mathcal{K}) / \mathcal{G}$ is (as an ideal of $\mathcal{A} / \mathcal{G}$ ) generated by its nontrivial projections of rank one (Proposition 3.14 and Theorem 3.15).

Structure of $(\mathcal{A} \cap \mathcal{K}) / \mathcal{G}$. To get a complete description of the structure of the ideal $(\mathcal{A} \cap \mathcal{K}) / \mathcal{G}$ in case $\mathcal{A}$ is a fractal algebra, we need the following result proved in [5], Theorem 4 .

THEOREM 3.21. Let $\mathfrak{B}$ be a unital $C^{*}$-algebra and $k$ a non-zero rank one element of $\mathfrak{B}$. Further let $\operatorname{Id}(k)$ denote the smallest closed ideal of $\mathfrak{B}$ which contains $k$. Then there exists an irreducible representation $(H, \pi)$ of $\mathfrak{B}$ such that

$$
\pi(\operatorname{Id}(k))=K(H) \quad \text { and } \quad \operatorname{Ker}\left(\left.\pi\right|_{\operatorname{Id}}(k)\right)=\{0\} .
$$

Consequently, if $k_{1}$ and $k_{2}$ are non-trivial rank one elements in a $C^{*}$ algebra $\mathfrak{B}$, then

$$
\text { either } \operatorname{Id}\left(k_{1}\right)=\operatorname{Id}\left(k_{2}\right) \quad \text { or } \quad \operatorname{Id}\left(k_{1}\right) \cap \operatorname{Id}\left(k_{2}\right)=\{0\} .
$$

This alternative suggests introducing an equivalence relation $\sim$ in the set of all non-trivial rank one elements in $(\mathcal{A} \cap \mathcal{K}) / \mathcal{G}$ as follows:

$$
k_{1} \sim k_{2} \quad \text { if } \operatorname{Id}\left(k_{1}\right)=\operatorname{Id}\left(k_{2}\right) .
$$

Let $T$ be the set of equivalence classes of this relation and, for each $t \in T$, let $I_{t}$ stand for the smallest closed ideal of $\mathcal{A} / \mathcal{G}$ which is generated by a non-trivial rank one element $k \in t$.

Theorem 3.22. Let $\mathcal{A}$ be a unital fractal $C^{*}$-subalgebra of $\mathcal{F}$ which contains the ideal $\mathcal{G}$.

(a) Each ideal $I_{t}$ is ${ }^{*}$-isometric to the ideal $K\left(H_{t}\right)$ of all compact operators on a Hilbert space $H_{t}$. $=\{0\}$.

(b) If $t_{1}, \ldots, t_{m} \in T$ and $t_{i} \neq t_{j}$ for $i \neq j$, then $\left(I_{t_{1}}+\ldots+I_{t_{m-1}}\right) \cap I_{t_{m}}$

(c) $(\mathcal{A} \cap \mathcal{K}) / \mathcal{G}$ is the smallest closed ideal of $\mathcal{A} / \mathcal{G}$ which contains every ideal $I_{t}$.

(d) There is a bijection between $T$ and the spectrum of $(\mathcal{A} \cap \mathcal{K}) / \mathcal{G}$.

Proof. Assertion (a) follows from Theorem 3.21, assertion (b) is proved in [5], and (c) is a consequence of (a) and of Theorem 3.20.

Now let $(H, W)$ be an irreducible representation of $(\mathcal{A} \cap \mathcal{K}) / \mathcal{G}$. Then there exists a non-trivial minimal projection $p \in(\mathcal{A} \cap \mathcal{K}) / \mathcal{G}$ such that $W$ does not vanish on $\operatorname{Id}(p)$ (otherwise $W$ would be the zero representation due to (c)). Hence, $\left.W\right|_{\operatorname{Id}(p)}$ is an irreducible representation of $\operatorname{Id}(p)$. Suppose 
that $W(q) \neq 0$ for another non-trivial minimal projection $q \not p$. Then $\operatorname{Id}(p) \cap \operatorname{Id}(q)=\{0\}$, i.e. $q k=k q=0$ for all $k \in \operatorname{Id}(p)$. Consequently,

$$
W(k) W(q)=W(k q)=0=W(q k)=W(q) W(k),
$$

i.e. $\operatorname{Im} W(q)$ is an invariant subspace for $W(\operatorname{Id}(p))$. Since $\left.W\right|_{\operatorname{Id}(p)}$ is an irreducible representation, this implies that either $\operatorname{Im} W(q)=\{0\}$ (which contradicts our assumption $W(q) \neq 0)$ or $\operatorname{Im} W(q)=H$. The latter is impossible if $\operatorname{dim} H \geq 2$, since $W(q)$ is an operator of rank one (cf. [5], Theorem 3). It remains to consider the case when $\operatorname{Im} W(q)=H$ and $\operatorname{dim} H=1$. Then, necessarily, $W(p)=1$ and $W(q)=1$, hence $W(p q)=1$ in contradiction to $p q=0$.

Thus, for every irreducible representation $(H, W)$ of $(\mathcal{A} \cap \mathcal{K}) / \mathcal{G}$, there is exactly one $t \in T$ such that $\left.W\right|_{I_{t}} \neq 0$. Conversely, since $I_{t} \cong K(H)$ by (a), it is evident that each ideal $I_{t}$ determines (up to unitary equivalence) exactly one irreducible representation $(H, W)$ of $(\mathcal{A} \cap \mathcal{K}) / \mathcal{G}$ with $\left.W\right|_{I_{t}} \neq 0$.

We mention one more consequence of the preceding theorem. Its proof is in [5], Theorems 5 and 6. For $t \in T$, we let $W_{t}$ denote the (essentially unique) irreducible representation from $I_{t}$ to the ideal $K\left(H_{t}\right)$; its existence is guaranteed by assertion (a) of the preceding theorem. The extensions of $W_{t}$ onto the algebras $\mathcal{A} / \mathcal{G}$ and $\mathcal{A}$ will again be denoted by $W_{t}$ for simplicity.

TheOREM 3.23 (Lifting theorem). Let $\mathcal{A}$ and $T$ be as before.

(a) An element $a \in \mathcal{A} / \mathcal{G}$ is invertible in $\mathcal{A} / \mathcal{G}$ if and only if the operators $W_{t}(a)$ are invertible in $L\left(H_{t}\right)$ for every $t \in T$ and if the coset $a+(\mathcal{A} \cap \mathcal{K}) / \mathcal{G}$ is invertible in the quotient algebra $(\mathcal{A} / \mathcal{G}) /((\mathcal{A} \cap \mathcal{K}) / \mathcal{G})$.

(b) Let $a \in \mathcal{A} / \mathcal{G}$ be invertible modulo $(\mathcal{A} \cap \mathcal{K}) / \mathcal{G}$. Then all operators $W_{t}(a)$ are Fredholm, and there are only finitely many $t \in T$ for which $W_{t}(a)$ is not invertible.

4. Fredholm theory in fractal algebras. Again let $\mathcal{A}$ be a unital fractal $C^{*}$-subalgebra of $\mathcal{F}$ which contains the ideal $\mathcal{G}$. The aim of this section is to specify the general Fredholm theory to the case of fractal algebras and to derive a formula for the $\alpha$-number of a fractal Fredholm sequence. Our starting point is the following result on the lifting of minimal projections.

Let $p \in(\mathcal{A} \cap \mathcal{K}) / \mathcal{G}$ be a non-trivial minimal projection. Then there is an essential projection $\left(\Pi_{n}\right) \in \mathcal{A} \cap \mathcal{K}$ which lifts $p$ (Corollary 3.12). We will see now that the behaviour of the sequence $\left(\operatorname{dim} \operatorname{Im} \Pi_{n}\right)$ for large $n$ depends only on the coset of $p$ with respect to the equivalence relation $\sim$.

THEOREM 4.1. Up to a sequence of integers which tends to zero, the sequence $\left(\operatorname{dim} \operatorname{Im} \Pi_{n}\right)_{n \geq 1}$ is uniquely defined by the coset of $p$ with respect to the equivalence relation $\sim$.

We prepare the proof by two simple lemmata. 
LEMMA 4.2. Let $\mathfrak{B}$ be a $C^{*}$-algebra, and let $p, q$ be rank one projections in $\mathfrak{B}$ with $q \in \operatorname{Id}(p)$. Then there is a $c \in \operatorname{Id}(p)$ such that $q=c^{*} p c$.

Proof. By Theorem 3.21, there is a ${ }^{*}$-isomorphism $W$ from $\operatorname{Id}(p)$ onto $K(H)$ for some Hilbert space $H$, and $W(p)$ is a non-zero projection of rank one on $H$ (see [5], Theorem 3). Thus, there is a $y \in H$ with $\|y\|=1$ such that

$$
W(p) x=\langle x, y\rangle y \quad \text { for all } x \in H .
$$

Analogously (recall that $\operatorname{Id}(p)=\operatorname{Id}(q)$ by $(19)$ ), there is a $z \in H$ with $\|z\|=1$ such that

$$
W(q) x=\langle x, z\rangle z \quad \text { for all } x \in H .
$$

Define $C \in K(H)$ by

$$
C x:=\langle x, z\rangle y \quad \text { for } x \in H .
$$

Then $C^{*} x=\langle x, y\rangle z$ and $C^{*} W(p) C x=W(q) x$ for all $x \in H$, as one easily checks. Since $W$ is a bijection, there is exactly one $c \in \operatorname{Id}(p)$ with $W(c)=C$, and this element $c$ satisfies $q=c^{*} p c$.

LEMma 4.3. Let $P$ and $Q$ be orthogonal projections on a Hilbert space $H$ and let $A, B \in L(H)$ be operators such that $\|Q-A P B\|<1$. Then

$$
\operatorname{dim} \operatorname{Im} Q \leq \operatorname{dim} \operatorname{Im} P .
$$

Proof. By (16), $\operatorname{dim} \operatorname{Im} Q=\operatorname{dim} \operatorname{Im} Q A P B Q$, whence the assertion.

Proof of Theorem 4.1. What we have to show is: If $p \sim q \in(\mathcal{A} \cap \mathcal{K}) / \mathcal{G}$ are non-trivial minimal projections with liftings $\left(\Pi_{n}^{p}\right)$ and $\left(\Pi_{n}^{q}\right)$, respectively, then

$$
\operatorname{dim} \operatorname{Im} \Pi_{n}^{p}=\operatorname{dim} \operatorname{Im} \Pi_{n}^{q} \quad \text { for all sufficiently large } n .
$$

First let $\left(\Pi_{n}^{1}\right)$ and $\left(\Pi_{n}^{2}\right)$ be liftings of one and the same minimal projection $p$. Then $\Pi_{n}^{1}=\Pi_{n}^{2}+G_{n}$ with a sequence $\left(G_{n}\right) \in \mathcal{G}$. From (16) we conclude that $\operatorname{dim} \operatorname{Im} \Pi_{n}^{1}=\operatorname{dim} \operatorname{Im} \Pi_{n}^{2}$ for all $n$ with $\left\|G_{n}\right\|<1$.

Let now $\left(\Pi_{n}^{p}\right)$ and $\left(\Pi_{n}^{q}\right)$ be liftings of $p \neq q$. By Lemma 4.2 , there is a $c \in \operatorname{Id}(p)=\operatorname{Id}(q)$ such that $q=c^{*} p c$. Let $\left(C_{n}\right) \in \mathcal{A} \cap \mathcal{K}$ be a lifting of $c$. Then, clearly,

$$
\Pi_{n}^{q}=C_{n}^{*} \Pi_{n}^{p} C_{n}+G_{n}
$$

with a sequence $\left(G_{n}\right) \in \mathcal{G}$. For $n$ sufficiently large, $\left\|G_{n}\right\|<1$, and for these $n$ we conclude from the preceding lemma that $\operatorname{dim} \operatorname{Im} \Pi_{n}^{q} \leq \operatorname{dim} \operatorname{Im} \Pi_{n}^{p}$. Now change the roles of $p$ and $q$ to get the reverse inequality.

Thus, with every $t \in T=\operatorname{Spec}(\mathcal{A} \cap \mathcal{K}) / \mathcal{G}$, we can associate a sequence $\left(\alpha_{n}^{t}\right)_{n \geq 1}$ by choosing a non-trivial minimal projection $p \in I_{t}$ with lifting $\left(\Pi_{n}^{p}\right)$ and by setting $\alpha_{n}^{t}:=\operatorname{dim} \operatorname{Im} \Pi_{n}^{p}$. Recall that this sequence is uniquely determined up to a sequence tending to zero. 
Let now $\mathbf{A}:=\left(A_{n}\right) \in \mathcal{A}$ be a Fredholm sequence, that is, $\left(A_{n}\right)$ is invertible modulo $\mathcal{K}$. Then the sequence $\mathbf{A}$ is also invertible modulo $\mathcal{A} \cap \mathcal{K}$, and from Theorem 3.23(b) we conclude that the operators $W_{t}(\mathbf{A})$ are Fredholm for every $t \in T$ and that only a finite number of them are not invertible. Thus, the following sums are actually finite:

$$
\alpha_{n}(\mathbf{A}):=\sum_{t \in T} \alpha_{n}^{t} \operatorname{dim} \operatorname{Ker} W_{t}(\mathbf{A})
$$

Evidently, (20) determines the sequence $\left(\alpha_{n}(\mathbf{A})\right)$ only up to a sequence tending to zero. The main result of the present paper is the following.

TheOREm 4.4. Let $\mathcal{A}$ be a unital fractal $C^{*}$-subalgebra of $\mathcal{F}$ which contains the ideal $\mathcal{G}$. If $\mathbf{A}=\left(A_{n}\right)$ is a Fredholm sequence in $\mathcal{A}$, then

$$
\lim _{n \rightarrow \infty} \sigma_{\alpha_{n}(\mathbf{A})}^{(n)}=0, \quad \liminf _{n \rightarrow \infty} \sigma_{\alpha_{n}(\mathbf{A})+1}^{(n)}>0
$$

where $0 \leq \sigma_{1}^{(n)} \leq \ldots \leq \sigma_{n}^{(n)}$ are the singular values of $A_{n}$.

Let us add a few comments on this result before its proof starts.

- For every Fredholm sequence $\mathbf{A}=\left(A_{n}\right) \in \mathcal{A}$, the number

$$
\alpha(\mathbf{A}):=\limsup _{n \rightarrow \infty} \alpha_{n}(\mathbf{A})
$$

is finite. Since $\left(\alpha_{n}(\mathbf{A})\right)$ is a sequence of non-negative integers, it has an infinite constant subsequence with entries equal to $\alpha(\mathbf{A})$. This shows that

$$
\liminf _{n \rightarrow \infty} \sigma_{\alpha(\mathbf{A})}^{(n)}=0, \quad \liminf _{n \rightarrow \infty} \sigma_{\alpha(\mathbf{A})+1}^{(n)}>0,
$$

i.e. the number $\alpha(\mathbf{A})$ defined by (22) is exactly the $\alpha$-number of the Fredholm sequence $\mathbf{A}$ as defined in Section 2. In that sense, one can consider (21) as an essential refinement of (10).

- The appearance of the limit in (21) shows that the singular values of a fractal Fredholm sequence $\left(A_{n}\right)$ have the splitting property, i.e. the singular values of $A_{n}$ are contained in $\left[0, \varepsilon_{n}\right] \cup[\delta, \infty)$ where $\varepsilon_{n} \rightarrow 0$ as $n \rightarrow \infty$ and where $\delta>0$. Thus, the coset $\left(A_{n}\right)+\mathcal{G}$ is Moore-Penrose invertible in $\mathcal{F} / \mathcal{G}$, which is in perfect accordance with the Moore-Penrose invertibility (or, equivalently, with the closedness of the range) of a Fredholm operator on a Hilbert space.

- Observe that the number of the singular values of $A_{n}$ which lie in $\left[0, \varepsilon_{n}\right]$ can depend on $n$ (it is just given by the number $\alpha_{n}(\mathbf{A})$ in $(21)$ ). It is not hard to construct fractal algebras with arbitrarily prescribed sequences $\left(\alpha_{n}^{t}\right)$. Consider, for example, for every $c \in \mathbb{C}$ and every $K \in K\left(l^{2}\right)$, the sequence $\left(A_{n}\right)$ with

$$
A_{n}:= \begin{cases}c P_{n}+P_{n} K P_{n} & \text { if } n \text { is even, } \\ c P_{n}+P_{n} K P_{n}+R_{n} K R_{n} & \text { if } n \text { is odd }\end{cases}
$$


where $P_{n}, R_{n}: l^{2} \rightarrow l^{2}$ are the operators mapping $\left(x_{1}, x_{2}, \ldots\right)$ to

$$
\left(x_{1}, \ldots, x_{n}, 0,0, \ldots\right) \text { and }\left(x_{n}, \ldots, x_{1}, 0,0, \ldots\right) \text {, }
$$

respectively. The smallest closed subalgebra $\mathcal{A}$ of $\mathcal{F}$ which contains all of these sequences is unital and symmetric, and it contains the ideal $\mathcal{G}$. Further, the mapping

$$
W: \mathcal{A} \rightarrow L\left(l^{2}\right), \quad\left(A_{n}\right) \mapsto \operatorname{s}-\lim A_{n} P_{n},
$$

is a representation of $\mathcal{A}$ which maps the sequence (24) to the operator $c I+K$. In particular, $W$ is an irreducible representation of $\mathcal{A}$. It is also easy to see that the invertibility of $c I+K$ implies the stability of the sequence (24). Since every sequence in $\mathcal{A}$ is a sum of a sequence of the form (24) and a sequence in $\mathcal{G}$, we conclude that a sequence $\left(A_{n}\right) \in \mathcal{A}$ is stable if and only if $W\left(A_{n}\right)$ is invertible. This shows that $\mathcal{A}$ is a fractal algebra and that the quotient algebra $\mathcal{A} / \mathcal{G}$ is ${ }^{*}$-isometric to the algebra $\mathbb{C} I+K\left(l^{2}\right)$.

Let $\left(\Pi_{n}\right)$ be the sequence of the form $(24)$ where $c=0$ and $K=P_{1}$. Then $p:=\left(\Pi_{n}\right)+\mathcal{G}$ is a non-trivial minimal projection in $(\mathcal{A} \cap \mathcal{K}) / \mathcal{G}$, and one has

$$
\operatorname{dim} \operatorname{Im} \Pi_{n}:= \begin{cases}1 & \text { if } n \text { is even, } \\ 2 & \text { if } n \text { is odd. }\end{cases}
$$

- In $[7,8]$, we exclusively considered algebras $\mathcal{A}$ of approximation sequences having the property that every non-trivial minimal projection in $(\mathcal{A} \cap \mathcal{K}) / \mathcal{G}$ lifts to a sequence of projections of rank one. Similarly, in [5], we studied the Fredholm theory for sequences in fractal algebras under the additional hypothesis that the ideal $\mathcal{A} \cap \mathcal{K}$ is generated by sequences of projections of rank one. Thus, in both cases, the numbers $\alpha_{n}^{t}$ are independent of $n$ and can be chosen to be 1 for all $n$. Under these assumptions, the formula (20) reduces to

$$
\alpha(\mathbf{A}):=\sum_{t \in T} \operatorname{dim} \operatorname{Ker} W_{t}(\mathbf{A})
$$

occurring in $[7,5]$.

The remainder of this section is devoted to the proof of Theorem 4.4. Once we derive the following proposition, the main steps in the proof of Theorem 4.4 will be as in [5].

Proposition 4.5. Let $q, r \in \mathcal{F} / \mathcal{G}$ be projections with $q r=r q=0$, and let $\left(R_{n}\right) \in \mathcal{F}$ be a lifting of $r$ (i.e. all matrices $R_{n}$ are orthogonal projections, and $\left.\left(R_{n}\right)+\mathcal{G}=r\right)$. Then there exists a projection $\left(Q_{n}\right)$ which lifts $q$ such that

$$
R_{n} Q_{n}=Q_{n} R_{n}=0 \quad \text { for all sufficiently large } n .
$$

Proof. Let $\left(Q_{n}^{\prime}\right)$ be a lifting of $q$ (which exists by Corollary 3.12) and consider the matrices $S_{n}:=\left(I_{n}-R_{n}\right) Q_{n}^{\prime}\left(I_{n}-R_{n}\right)$. The coset modulo $\mathcal{G}$ of 
the sequence $\left(S_{n}\right)$ is

$$
(e-r) q(e-r)=q-r q-q r+r q r=q .
$$

Hence $((e-r) q(e-r))^{2}=(e-r) q(e-r)$, i.e. $\left\|S_{n}-S_{n}^{2}\right\| \rightarrow 0$. Choose $n_{0}$ such that $\left\|S_{n}-S_{n}^{2}\right\|<1 / 4$ for all $n \geq n_{0}$. Then, by Proposition 3 of [7], for all $n \geq n_{0}$ there is a $G_{n}$ which belongs to the $C^{*}$-algebra generated by $I_{n}-R_{n}$ and $S_{n}$ such that $S_{n}+G_{n}$ is a projection and

$$
\left\|G_{n}\right\| \leq 2\left\|S_{n}-S_{n}^{2}\right\| \text {. }
$$

Define $Q_{n}:=Q_{n}^{\prime}$ if $n<n_{0}$ and $Q_{n}:=S_{n}+G_{n}$ if $n \geq n_{0}$. Then, for $n \geq n_{0}$,

$$
\begin{aligned}
\left\|Q_{n}-Q_{n}^{\prime}\right\| & =\left\|S_{n}-Q_{n}^{\prime}+G_{n}\right\| \\
& \leq\left\|\left(I_{n}-R_{n}\right) Q_{n}^{\prime}\left(I_{n}-R_{n}\right)-Q_{n}^{\prime}\right\|+\left\|G_{n}\right\| \rightarrow 0
\end{aligned}
$$

because of $(26)$. Hence, $\left(Q_{n}\right)$ is also a lifting of $q$, and this lifting satisfies (25):

$$
R_{n} Q_{n}=R_{n}\left(I_{n}-R_{n}\right) Q_{n}^{\prime}\left(I_{n}-R_{n}\right)+R_{n} G_{n}=0
$$

(recall that $R_{n} G_{n}=0$ since $G_{n} \in \operatorname{alg}\left(I_{n}-R_{n}, S_{n}\right)$ ).

Let now $\left(A_{n}\right) \in \mathcal{A}$ be a Fredholm sequence, i.e. $\left(A_{n}\right)$ is invertible modulo the ideal $\mathcal{A} \cap \mathcal{K}$. Then, by Theorem 3.23, the operators $W_{t}\left(\left(A_{n}\right)\right)$ are Fredholm for every $t \in T$ and only finitely many of them are not invertible. Let $P_{\text {Ker } W_{t}\left(\left(A_{n}\right)\right)}$ denote the orthogonal projection from $H_{t}$ onto the kernel of $W_{t}\left(\left(A_{n}\right)\right.$ ) (only a finite number of these projections is not zero). Decompose each of these projections into a sum of $\operatorname{dim} \operatorname{Ker} W_{t}\left(\left(A_{n}\right)\right)$ orthogonal projections of rank one:

$$
P_{\text {Ker } W_{t}\left(\left(A_{n}\right)\right)}=\sum_{i=1}^{\operatorname{dim} \operatorname{Ker} W_{t}\left(\left(A_{n}\right)\right)} P_{i, t}
$$

such that $P_{i, t} P_{j, t}=P_{j, t} P_{i, t}=0$ whenever $i \neq j$ (for example, by choosing an orthonormal basis in $\operatorname{Ker} W_{t}\left(\left(A_{n}\right)\right)$ and by defining $P_{i, t}$ as the orthogonal projection onto the $i$ th element of this basis). Since $W_{t}$ is an isomorphism between $I_{t}$ and $K\left(H_{t}\right)$, every projection $P_{i, t}$ corresponds uniquely to a coset $p_{i, t} \in I_{t}$. Clearly, $p_{i, t} p_{j, t}=p_{j, t} p_{i, t}=0$ if $i \neq j$. Since $I_{s} \cap I_{t}=\{0\}$ for all $s, t \in T$ with $s \neq t$, one has moreover

$$
p_{i, t} p_{j, s}=p_{j, s} p_{i, t}=0 \quad \text { whenever }(i, t) \neq(j, s) .
$$

Using Proposition 4.5 it is not hard to see that the cosets $p_{i, t}$ can be lifted to sequences $\left(P_{n}^{i, t}\right)$ in such a way that

$$
P_{n}^{i, t} P_{n}^{j, s}=P_{n}^{j, s} P_{n}^{i, t}=0 \quad \text { whenever }(i, t) \neq(j, s)
$$

for all sufficiently large $n$. Indeed, suppose we have already lifted a finite number of the projections $p_{i, t}$ in such a way that $(28)$ holds. Let $\left(R_{n}\right)$ be the sum of these liftings. Then every $R_{n}$ with $n$ large enough is an orthogonal 
projection due to (28). If $q$ is an element of the family of the projections $p_{i, t}$ which has not yet been lifted, then we construct a lifting $\left(Q_{n}\right)$ of $q$ such that $R_{n} Q_{n}=Q_{n} R_{n}=0$ for all sufficiently large $n$, which is possible due to Proposition 4.5. It is obvious that the new set of all liftings (enlarged by $\left(Q_{n}\right)$ ) again satisfies the condition (28). This procedure terminates since there are only a finite number of projections to lift.

The orthogonality (28) ensures that the operators

$$
R_{n}:=\sum_{t \in T} \sum_{i=1}^{\operatorname{dim} \operatorname{Ker} W_{t}\left(\left(A_{n}\right)\right)} P_{n}^{i, t}
$$

are orthogonal projections for all sufficiently large $n$ and that

$$
\begin{aligned}
\operatorname{dim} \operatorname{Im} R_{n} & =\sum_{t \in T} \sum_{i=1}^{\operatorname{dim} \operatorname{Ker} W_{t}\left(\left(A_{n}\right)\right)} \alpha_{n}^{t}=\sum_{t \in T} \alpha_{n}^{t} \operatorname{dim} \operatorname{Ker} W_{t}(\mathbf{A}) \\
& =\alpha_{n}(\mathbf{A})
\end{aligned}
$$

with $\mathbf{A}=\left(A_{n}\right)$ for all sufficiently large $n$. As in [5] one can now easily check that

$$
\left(A_{n}\right)^{*}\left(A_{n}\right)+\left(R_{n}\right) \quad \text { is a stable sequence and }\left(A_{n}\right)^{*}\left(A_{n}\right)\left(R_{n}\right) \in \mathcal{G} .
$$

Thus, the coset $\left(A_{n}\right)+\mathcal{G}$ is Moore-Penrose invertible in $\mathcal{A} / \mathcal{G}$, and $\left(R_{n}\right)+\mathcal{G}$ is the associated Moore-Penrose projection. It is shown in Theorem 3 of [7] that there is then a sequence $\left(\Pi_{n}\right)$ of orthogonal projections such that $\left\|\Pi_{n}-R_{n}\right\| \rightarrow 0$ and that every projection $\Pi_{n}$ belongs to the $C^{*}$-algebra generated by $A_{n}^{*} A_{n}$ and by the identity matrix $I_{n}$. From $\left\|\Pi_{n}-R_{n}\right\| \rightarrow 0$ we conclude that

$$
\operatorname{dim} \operatorname{Im} \Pi_{n}=\operatorname{dim} \operatorname{Im} R_{n}=\alpha_{n}(\mathbf{A}) \quad \text { for all sufficiently large } n,
$$

and the property $\Pi_{n} \in \operatorname{alg}\left(A_{n}^{*} A_{n}, I_{n}\right)$ ensures that the matrices $A_{n}^{*} A_{n}$ and $\Pi_{n}$ can be diagonalized simultaneously:

$$
\begin{aligned}
U_{n}^{*} A_{n}^{*} A_{n} U_{n} & =\operatorname{diag}\left(\lambda_{1}^{(n)}, \ldots, \lambda_{n}^{(n)}\right), \\
U_{n}^{*} \Pi_{n} U_{n} & =\operatorname{diag}\left(\pi_{1}^{(n)}, \ldots, \pi_{n}^{(n)}\right)
\end{aligned}
$$

with unitary matrices $U_{n}$ which we choose in such a way that

$$
0 \leq \lambda_{1}^{(n)} \leq \ldots \leq \lambda_{n}^{(n)} .
$$

The conditions (30) (with $\Pi_{n}$ in place of $R_{n}$ ) imply the existence of a $C>0$ such that

$$
\begin{aligned}
\lambda_{i}^{(n)}+\pi_{i}^{(n)} \geq C>0 & \text { for all } n \geq n_{0} \text { and } i=1, \ldots, n, \\
\lambda_{i}^{(n)} \cdot \pi_{i}^{(n)}<C & \text { for all } n \geq n_{0} \text { and } i=1, \ldots, n,
\end{aligned}
$$


Let $i=i_{n}$ be such that

$$
\lambda_{1}^{(n)} \leq \ldots \leq \lambda_{i}^{(n)}<c \leq \lambda_{i+1}^{(n)} \leq \ldots \leq \lambda_{n}^{(n)} .
$$

Then, for (31) to hold it is necessary that

$$
\pi_{1}^{(n)}=\ldots=\pi_{i}^{(n)}=1
$$

whereas (32) requires that

$$
\pi_{i+1}^{(n)}=\ldots=\lambda_{n}^{(n)}=0
$$

Hence, $i_{n}=\alpha_{n}(\mathbf{A})$ and

$$
U_{n}^{*} \Pi_{n} U_{n}=\operatorname{diag}(1, \ldots, 1,0, \ldots, 0)
$$

with $\alpha_{n}(\mathbf{A})$ ones and $n-\alpha_{n}(\mathbf{A})$ zeros. Now the identity (21) follows from the diagonalized form of (30) with $\Pi_{n}$ in place of $R_{n}$. This finishes the proof of Theorem 4.4

\section{References}

[1] A. Böttcher and B. Silbermann, Introduction to Large Truncated Toeplitz Matrices, Springer, New York, 1999.

[2] R. Hagen, S. Roch and B. Silbermann, $C^{*}$-Algebras and Numerical Analysis, Dekker, New York, 2000.

[3] G. K. Pedersen, $C^{*}$-Algebras and Their Automorphism Groups, Academic Press, London, 1979.

[4] S. Roch, Algebras of approximation sequences: Fractality, in: Problems and Methods in Mathematical Physics (Chemnitz, 1999), Oper. Theory Adv. Appl. 121, Birkhäuser, 2001, 471-497.

[5] S. Roch, Algebras of approximation sequences: Fredholmness, preprint 2048(1999) TU Darmstadt, submitted to: J. Oper. Theory.

[6] S. Roch and B. Silbermann, $C^{*}$-algebra techniques in numerical analysis, J. Oper. Theory 35 (1996), 241-280.

[7] - , - Index calculus for approximation methods and singular value decomposition, J. Math. Anal. Appl. 225 (1998), 401-426.

[8] —, - A note on singular values of Cauchy-Toeplitz matrices, Linear Algebra Appl. 275-276 (1998), 531-536.

Fachbereich Mathematik

Technische Universität Darmstadt

Schlossgartenstrasse 7

64289 Darmstadt, Germany

E-mail: roch@mathematik.tu-darmstadt.de 